\title{
Volumetric Arc Therapy Seems More Promising for Sparing Organs at Risk in Adjuvant Postoperative Radiotherapy for Pancreatic Adenocarcinoma than Step-and-Shoot Intensity-Modulated Radiotherapy
}

\author{
Yasemin BÖLÜKBAŞI, ${ }^{1,2,3}$ Yücel SAĞLAM, ${ }^{2}$ Duygu SEZEN, ${ }^{1}$ \\ Z. Vildan ALPAN, ${ }^{2}$ Steve KIRSNER, ${ }^{3}$ Uğur SELEK ${ }^{1,2,3}$ \\ 'Department of Radiation Oncology, Koç University Faculty of Medicine, Istanbul-Turkey \\ ${ }^{2}$ Department of Radiation Oncology, American Hospital-MD Anderson Radiation Treatment Center, İstanbul-Turkey \\ ${ }^{3}$ Department of Radiation Oncology, MD Anderson Cancer Center, Houston-USA
}

\begin{abstract}
OBJECTIVE
The aim of the present study was to determine if volumetric modulated arc therapy (VMAT) provided superior dose distribution than intensity-modulated radiotherapy (step-and-shoot; ssIMRT) based on target volume coverage and organs at risk (OARs) doses in postoperative radiotherapy for pancreatic cancer patients.
\end{abstract}

\section{METHODS}

New 4-dimensional computed tomography plans for 10 pancreatic cancer patients were created. The ssIMRT plans had 6 coplanar fields $\left(330-0-30-60-90^{\circ}\right)$ and VMAT plans were generated with $2268-92^{\circ}$ arcs.

\section{RESULTS}

VMAT plans revealed better overall sparing of right kidney (volume receiving 15\% of prescribed dose [V15]: $28.3 \%$ vs $46.9 \%$, $\mathrm{p}=0.012$; V20: $16.1 \%$ vs $27.6 \%$, $\mathrm{p}=0.007$; V25: $8.6 \%$ vs $15.2 \%$, $\mathrm{p}=0.005$; mean dose 1549 centigray [cGy] vs $1987 \mathrm{cGy}, \mathrm{p}=0.005$ ). VMAT delivered similar isodose distribution (planning target volume [PTV] mean dose: 5164 vs 5183 cGy, PTV max: 5526 cGy vs 5505 cGy; p=0.541) with significantly fewer monitor units (MU) (MU: 468 vs 527; $\mathrm{p}=0.032$ ) in comparison with ssIMRT. VMAT was also found to be superior for V30 intestinal dose, but mean dose was similar (1963 cGy vs 2032 cGy; p=0.05).

\section{CONCLUSION}

VMAT provided more effective protection for bilateral kidneys and small intestine with better OAR doses, as well as for liver, with reduced high-dose volumes in this cohort. This could be investigated as more tolerable concurrent radiochemotherapy treatment with better OAR preservation.

Keywords: Intensity-modulated radiation therapy; pancreatic cancer; volumetric modulated arc therapy.

Copyright $\odot$ 2017, Turkish Society for Radiation Oncology

\section{Introduction}

Pancreas cancer is named as one of the most complex cancer to treat, and even if surgical resection is per- formed, the recurrence rate is high and the survival rate after surgery is poor. There are two different approaches in worldwide such as "chemoradiotherapy followed by chemotherapy" is considered the optimal 
therapy in the United States, while "chemotherapy alone" is the current standard in Europe.[1] One of the important reason for this divergence especially in the postoperative setting, is the location of pancreas as it is located in the middle of mutiple critical structures like kidneys, small bowel, liver and spinal cord which leads more radiotherapy toxicity.[2]

Intensity-modulated radiation therapy (IMRT) is an advanced mode of high-precision radiotherapy that delivers radiation doses precisely to the threedimensional shape of the tumor by modulating the intensity of the radiation beam in multiple small volumes while minimizing the dose to surrounding normal critical structures. [3] In the postoperative or curative setting, compared to 3-D Conformal planning, IMRT has encouraging gastrointestinal toxicity results in treating pancreas cancer without compromising local control.[4-8] In the dosimetric evaluation by Landry et al., compared to 3D-CRT, using IMRT reduced the amount of small bowel receiving more than 50 Gy form $31 \%$ to 19.2\%.[5] First clinical result from Wayne state University reported 7\% incidence of grade 3 gastrointestinal toxicity and one year actuarial survival rate of $69 \%$ for postoperative or locally advanced pancreas cancer patients treated with 54-55 Gy IMRT.[6] Systematic review of clinical results comparing IMRT to 3DCRT have also shown the reduction of grade 2 and 3 toxicities without compromising of local control.[9]

The volumetric modulated arc therapy (VMAT) technique is a recent form of IMRT with using one or two-arc gantry rotation by simultaneously modulating the MLC position and the dose rates. In the recent reports, VMAT has been shown to be superior for a variety of cancer types such as head and neck, prostate cancer and the dosimetric and clinic publications seeking for new cancer sites to use VMAT were increasing. [10,11] As the motion is an important problem in radiotherapy planning of pancreas cancer, VMAT can decrease the risk of intrafractional organ motion by shortening treatment time.[12] In the dosimetric studies, comparing VMAT to Helical tomotherapy, IMRT and 3D-CRT, reduced organ at risk doses such as kidney and liver have a promising effect on reducing toxicity due to radiotherapy. $[13,14]$

We aimed to define whether VMAT provides a superior dose distribution in comparison to Intensity modulated radiotherapy (Step and shoot: ssIMRT) based on 4D-CT target volume coverage and organs at risk (OAR) doses in adjuvant postoperative radiotherapy for pancreas adenocarcinoma patients.

\section{Materials and Methods}

\section{Patients}

We have re-planned the planning 4D CT (Respiratory data sets are "binned" by phase: $0-100 \%$ at $10 \%$ interval) scans of our ten consecutive stage IIB pancreas cancer patients in 2011. All patients were treated with postoperative concurrent radiochemotherapy of 50.4 Gy in 28 fractions (ssIMRT, 1.8 Gy/fraction/day) with Capecitabine $2500 \mathrm{mg} / \mathrm{m}^{2}$. Whipple surgery for pancreatic head tumors was performed.

\section{Simulation and target contouring}

All the patients were simulated in the supine position on a customized vacuum bed, with using T-bar, Wingboard, and knee-foot stopper. 4-D and contrast freebreathing axial CT scans with $3 \mathrm{~mm}$ slice thickness with were obtained by AcQSim CT simulation of Philips Brilliance Big Bore CT. Respiratory correlated imaging was generated for planning which was performed by Pinnacle radiation therapy planning system (9.0, Philips Medical Systems Inc. Cleveland, $\mathrm{OH}$ ) which uses Collapse Cone [cc] convolution algorithm. Also for every patient a second scan with intravenous contrast were obtained and fused for contouring purposes.

\section{Planning}

Clinical target volume (CTV) was delineated according to departmental guidelines and RTOG pancreas cancer web based CT contouring atlas,[8] as porta hepatis, superior mesenteric artery, celiac, paraaortic and postoperative bed including peripancreatic nodal area based on preoperative CT fusion. An internal CTV as ITV, showing the position of CTV in all 4D-CT scans were generated by one physician and the second physician reviewed all the contours. Planning target volume (PTV) was integrated tumor volume (ITV=internal CTV contoured on all respiratory data sets) plus $4 \mathrm{~mm}$. A "small-bowel region" was defined which consisted of the abdominal content after subtracting the PTV, all OARs and the vertebral bodies, with the posterior border extending to the dorsum of the lumbar vertebral body, but excluding the retroperitoneal space.[12] Liver was delineated at MinIP phase.

The prescribed dose was 50.4 Gy in 28 fractions and the planning objective was to give at least $95 \%$ prescribed dose to PTV and 98\% prescribed doses to CTV. Identical objectives were used for IMRT and VMAT plans. The planning objectives were selected as listed; The maximum point dose to spinal cord is less than 45 Gy, volume of kidney receiving more than 20 Gy (V20) 
Table 1 Dosimetric comparisons of IMRT and VMAT planning techniques for postoperative pancreas cancer patients

\begin{tabular}{|c|c|c|c|c|c|}
\hline Parameter & VMAT mean dose & VMAT range & ssIMRT mean & ssIMRT range & $\mathbf{p}$ \\
\hline \multicolumn{6}{|l|}{ Left kidney } \\
\hline V15 (\%) & 23.4 & $4.8-41$ & 29.8 & $7.3-56$. & 0.74 \\
\hline V20 (\%) & 11.6 & $1.5-30$ & 18.8 & $3.2-32$ & 0.018 \\
\hline V25 (\%) & 5.7 & $0.3-14$ & 11.7 & $1.3-24$ & 0.01 \\
\hline Mean dose (cGy) & 1155 & $695-1617$ & 1209 & $808-1771$ & 0.33 \\
\hline \multicolumn{6}{|l|}{ Right kidney } \\
\hline V15 (\%) & 28.3 & $9-49$ & 46.9 & $28-62$ & 0.012 \\
\hline V20 (\%) & 16.1 & $5-30$ & 27.6 & 19-39 & 0.007 \\
\hline V25 (\%) & 8.6 & $1-18$ & 15.2 & $9-27$ & 0.005 \\
\hline Mean dose (cGy) & 1549 & $811-3832$ & 1987 & $1300-4853$ & 0.005 \\
\hline \multicolumn{6}{|l|}{ Liver } \\
\hline V30 (\%) & 18.3 & $10-30$ & 20.1 & $12-30$ & 0.012 \\
\hline V45 (\%) & 7.9 & $5-14$ & 9.5 & $6-15$ & 0.006 \\
\hline Mean dose (cGy) & 1507 & 1094-2320 & 1550 & $1097-2113$ & 0.168 \\
\hline \multicolumn{6}{|l|}{ Small bowel } \\
\hline V30 (\%) & 28.1 & $19-48$ & 32.9 & $22-59$ & 0.008 \\
\hline Mean dose (cGy) & 1963 & 1009-2963 & 2032 & $1017-3278$ & 0.05 \\
\hline Spinal cord maximum dose (cGy) & 3697 & $3199-4100$ & 3792 & $3456-4233$ & 0.24 \\
\hline PTV mean dose (cGy) & 5183 & $5097-5260$ & 5164 & $5096-5195$ & 0.06 \\
\hline PTV maximum dose (cGy) & 5505 & $5370-5971$ & 5526 & $5325-5973$ & 0.541 \\
\hline Monitor units & 468 & $319-624$ & 527 & $420-583$ & 0.032 \\
\hline
\end{tabular}

SSIMRT: Step and shoot intensity modulated radiotherapy; VMAT: Volumetric modulated arc radiotherapy.

$<33 \%$, if one kidney exceeds the above, then spare the other kidney with $20 \mathrm{~Gy}(\mathrm{~V} 20)<20 \%$, mean liver dose (MLD) $<32 \mathrm{~Gy}, \mathrm{~V} 20 \mathrm{~Gy}<66 \%$.

Treated ssIMRT plans were 6 coplanar fields (3300-30-60-90 degree) with multiple segments. VMAT plans were generated as two $268-92^{\circ}$ arcs rotating clockwise and counter clockwise starting from $92^{\circ}$ and $268^{\circ}$ with $15^{\circ}$ collimator angle. Collimator angle was fixed to $-15^{\circ}$ to minimize the effects of interleaf leakage and tongue-and groove effect. For all the plans, $6 \mathrm{MV}$ Photon beams created by using Varian Linac Triology (Rapid-Arc) 120 leaf millennium multileaf collimator (MLC) with a maximum dose rate $600 \mathrm{MU} / \mathrm{min}$ and Grid Size was $0.3 \times 0.3 \times 0.3 \mathrm{~cm}$ for both plan calculations. All plans were performed by one physicist (YS).

\section{Comparison of VMAT and ssIMRT techniques}

The maximum dose (Gy) for spinal cord, V15-V20-V25 for bilateral kidney, V30 and mean dose for intestine, V30-V45 for liver, total treated monitor units (MU), and mean and maximum doses for PTV were compared based on dose volume histograms. VMAT and ssIMRT techniques were compared using two-tailed pair wise Wilcoxon signed-ranked tests, with $\mathrm{p}<0.05$ considered to indicate statistically significant differences.

\section{Results}

The mean ITV volume in 10 patients was $462.0 \mathrm{~cm}^{3}$ (range: $286.8-793.5 \mathrm{~cm}^{3}$ ). The mean doses to liver, both kidneys and other critical structures were listed in Table 1.

\section{Target}

In all the plans PTV was covered at least 95\% prescribed dose of 50.4Gy. VMAT delivered similar isodose distrubutions (PTV mean dose: 5164 vs 5183 cGy, PTV Max 5526 cGy vs 5505 cGy, p=0.541). As an advantage VMAT has been delivered by significantly less MU (MU: 468 vs 527, $\mathrm{p}=0.032$ ) in comparison to ssIMRT. Representative isodose curves for ssIMRT and VMAT technique were given in axial, sagittal and coronal CT slices at Figures 1-3.

Kidneys; Right kidney was spared better as V15$28.3 \%$ vs $46.9 \%, \mathrm{p}=0.012$; V20- $16.1 \%$ vs $27.6 \%$, $\mathrm{p}=0.007$; V25- $8.6 \%$ vs $15.2 \%$, $\mathrm{p}=0.005$; mean dose $1549 \mathrm{cGy}$ vs $1987 \mathrm{cGy}, \mathrm{p}=0.005$ compared to 6 field ssIMRT. For left kidney, there was decrease in high dose regions (V20 $11.6 \%$ vs $18.8 \%$; $\mathrm{p}=0.008$, V25 5.7\% vs $11.7 \%$; $\mathrm{p}=0.018$ ). No statistical difference was found in terms of mean doses (1155 cGy vs 1209 cGy, p=0.33) and V15 -low dose area (V15; 23.4\% vs 29.8\%, $\mathrm{p}=0.74)$ for left kidney. 

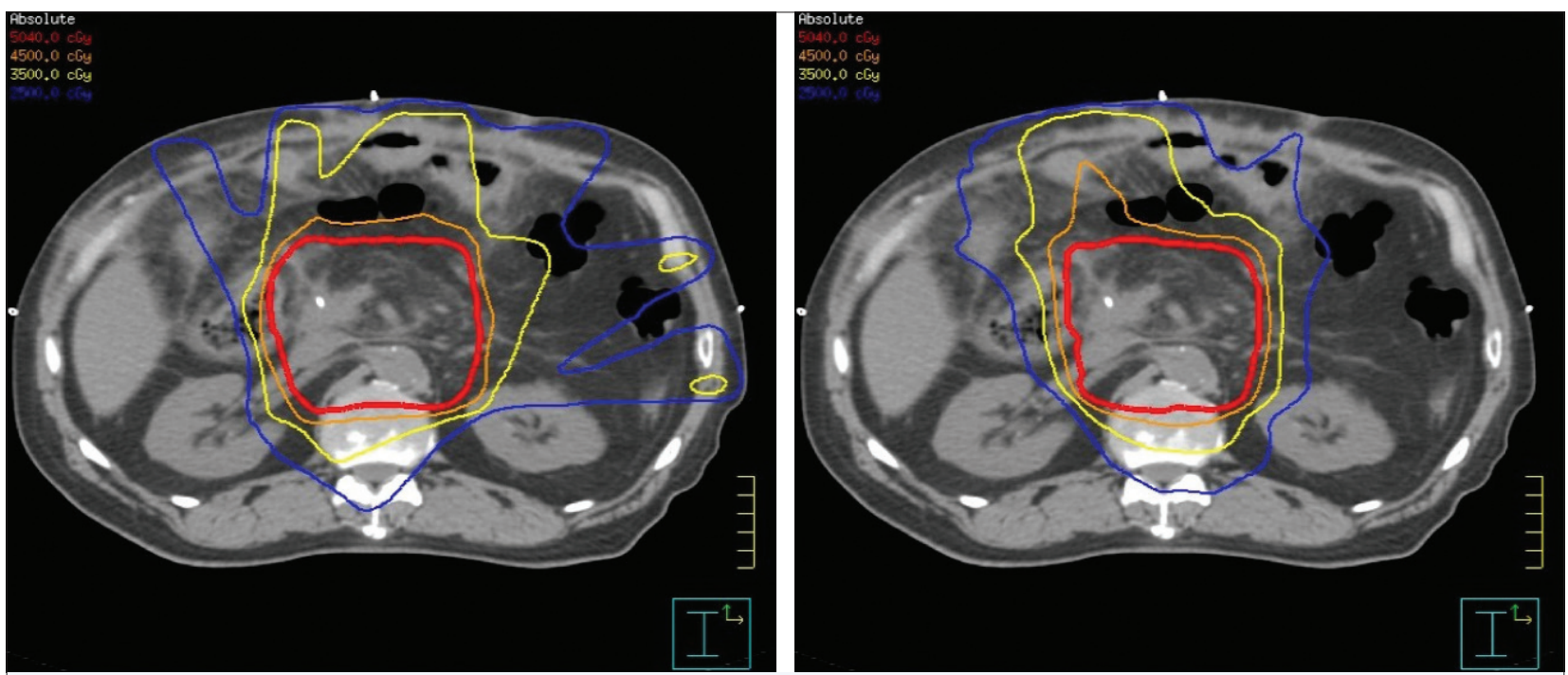

Fig. 1. Representative isodose curves for step and shoot intensity modulated radiotherapy (ssIMRT) and volumetric arc therapy (VMAT) technique. 54 Gy curve-red, 45 Gy curve-orange 35 Gy curve-yellow and 25 Gy curve-blue were shown in axial slices.
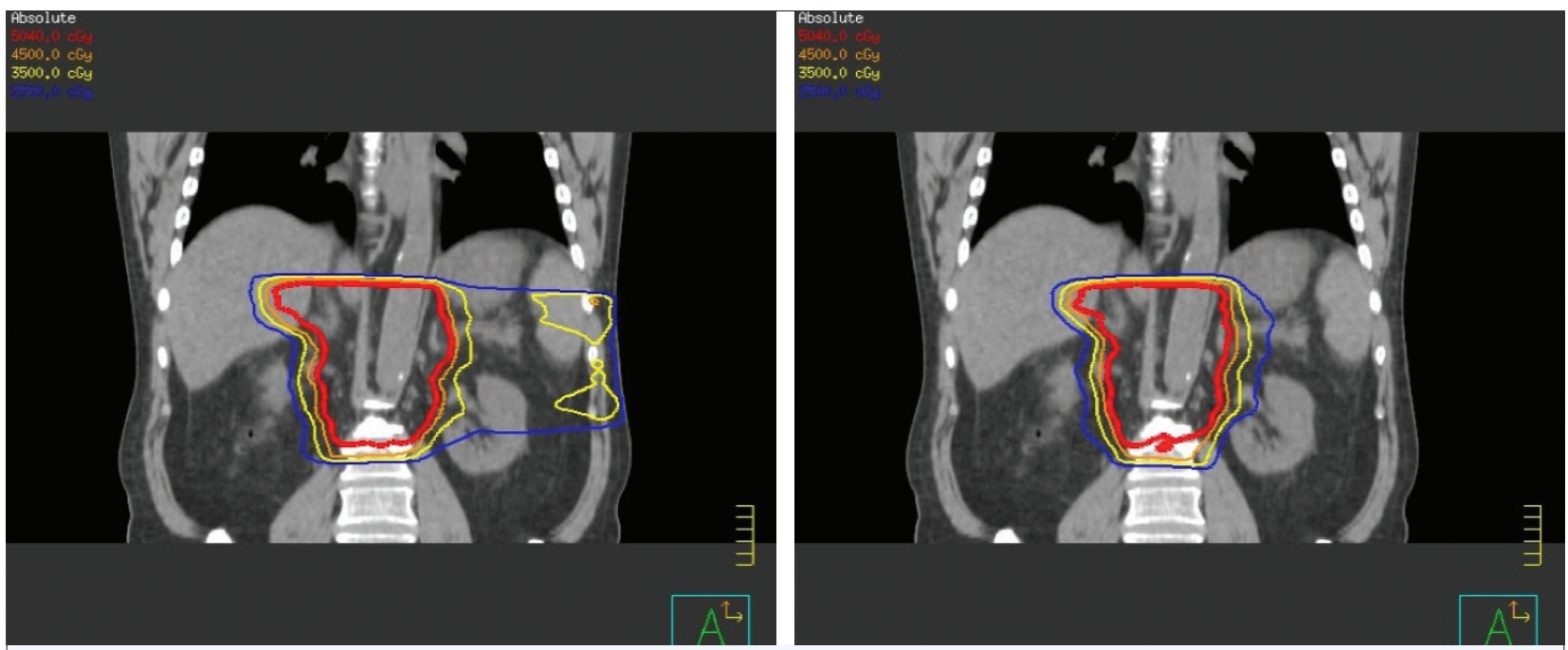

Fig. 2. Representative isodose curves for step and shoot intensity modulated radiotherapy (ssIMRT) and volumetric arc therapy (VMAT) technique. 54 Gy curve-red, 45 Gy curve-orange 35 Gy curve-yellow and 25 Gy curve-blue were shown in coronal slices.

Small Bowel; VMAT was also found to be superior for intestinal doses (V30: 28.1\% vs 32.9\%, p=0.008) but both planning technique provides similar mean doses of small bowel.

Liver; the mean doses were found to be similar in each plan, but high dose volumes such as V30 and V45 were decreased by VMAT (VMAT vs ssIMRT: mean dose- 1507 cGy vs 1550 , $\mathrm{p}=0.168$, V30- $18.3 \%$ vs $20.1 \%$, $\mathrm{p}=0.012$, V45- $7.9 \%$ vs $9.5 \%, \mathrm{p}=0.006$ ).

Spinal cord; Both plans provided acceptable and comparable spinal cord doses (cord maximum dose 3792 cGy vs 3697 cGy, p=0.24).

\section{Discussion}

Radiotherapy for pancreas cancer is a challenging area due to its location and motion factor because of respiratory and other organ movements. In attempts to dose escalation in the adjuvant setting, Allen et al., reported that maximum tolerated dose was 39 Gy using 3DCRT 

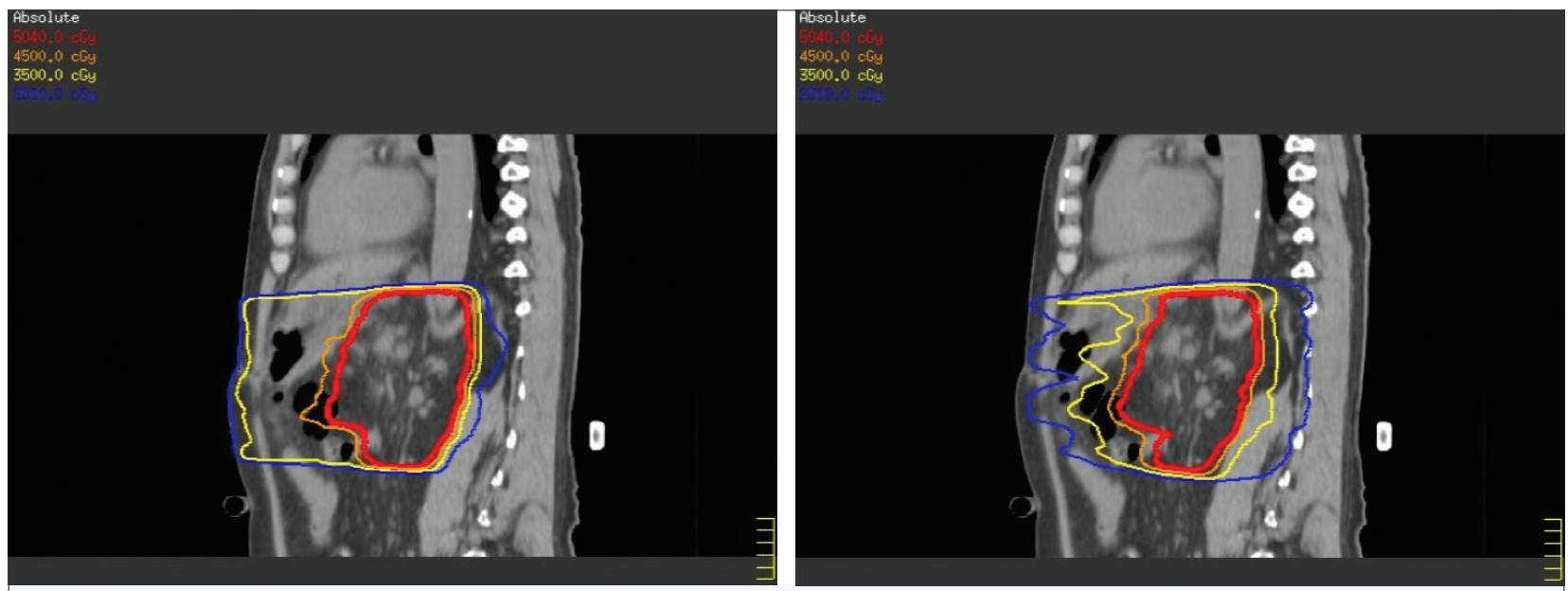

Fig. 3. Representative isodose curves for step and shoot intensity modulated radiotherapy (ssIMRT) and Volumetric arc therapy (VMAT) technique. 54 Gy curve-red, 45 Gy curve-orange 35 Gy curve-yellow and 25 Gy curve-blue were shown in sagittal slices.

with concurrent gemcitabine, and escalation to $42 \mathrm{~Gy}$ resulted increased gastrointestinal toxicity.[15] Also the gastrointestinal tumor study group (GITSG) trial that lead the treatment approach used today, delivered 40 Gy split course radiotherapy with a 2 weeks break.[16] IMRT has been shown to improve dosimetric parameters and decrease normal tissue doses; as a result it has been accepted as a routine approach. The first important problem of radiotherapy is the high rated of gastrointestinal acute side effects during concurrent radiotherapy. While older series using 3DCRT has almost 58\% nonhematological grade 3 or more acute toxicity rate, newer series using IMRT has a rate ranging from $7 \%$ to $16 \%$. $[6,16-18]$ The second concern was interfraction and intrafraction movement in pancreas cancer, using VMAT which is a novel form of IMRT which offers fast and homogeneous dose delivery and 4DCT simulation, could provide more effective dose distribution and achieve to maintain the dose delivery as it was planned. In our study, based on 4DCT scans, VMAT allowed dose reduction in right kidney, liver, small bowel and high dose volume of left kidney compared to ssIMRT. To our knowledge, this is the first study that compares VMAT to IMRT based on $4 \mathrm{D}$-CT scans of a pure patient population whom underwent Whipple operation.

One of the important acute side effect was caused by the dose that small bowel received during therapy. It is shown that even large volume of low dose areas (5-15 Gy) also can cause toxicity.[19] This is a concern with using novel technologies, this is not supported by clinical studies evaluating acute GI side effects.[18] Landry et al., delivered 45 Gy for gross tumor volume and micro- scopic disease, and in comparison with 3DCRT, IMRT decreased the dose that one third of the small bowel receiving from 38.5 Gy vs. 30.2 Gy. Also IMRT reduced the median volume of small bowel receiving more than 50 Gy.[5] Milano et al., analyzed 25 patients who was treated for pancreas and bile duct cancers with concurrent chemoradiotherapy using IMRT technique. Of the patients, $80 \%$ has grade 2 or less acute upper GI toxicity. Memorial Sloan Kettering Cancer center has evaluated 205 patients that were treated for locally advanced pancreas cancer undergoing IMRT or 3DCRT. The evaluation of Grade 2+ GI toxicity revealed a reduction from $34 \%$ to $16 \%$ by using IMRT compared to using 3DCRT which shows the clinical benefit of using advanced planning.[4] There results were supported by a systematic review of published clinical studies consisting 45 patients comparing IMRT and 3DCRT where grade 3 toxicities were dropped from $10.6 \%$ to $5 \%(\mathrm{p}=0.017)$. In our study VMAT was also found to be superior to IMRT for high dose region (V30) but no significant difference for the mean small bowel doses with using 4DCT planning.

Brown et al., compared IMRT, integrated boost IMRT and 3DCRT for locally advanced pancreas cancer and revealed that left kidney was spared better in 3DCRT due to the use of multiple fields in IMRT, in contrast to the right kidney doses which was better with IMRT.[7] In other comparison of IMRT to 3DCRT, V20 right kidney doses were lowered from $27.7 \%$ to $16 \%$, but this not observed for left kidney (5.7\% vs $11.1 \%, p=0.1)$.[20] The superiority of IMRT over 3DCRT has been also shown dosimetrically by Bahl et al., where V45 for bowel bag was $212.3 \pm 159.0 \mathrm{cc}$ (mean volume \pm standard deviation) 
versus $80.9 \pm 57.4 \mathrm{cc}$ in 3 DCRT versus IMRT $(\mathrm{p}=0.033)$, and V28 dose for bilateral kidneys was $32.7 \pm 23.5 \mathrm{cc}$ (mean volume \pm standard deviation) versus $7.9 \pm 7.4 \mathrm{cc}$ for 3DCRT versus IMRT, respectively $(\mathrm{p}=0.013)$.[21] These differences can be caused by the design of the fields and dose constraints that were used for advanced planning. In the recent years, VMAT has been compared to other techniques. Compared to $3 \mathrm{D}$ planning of pancreatic and bile duct malignancies, VMAT plans found to be superior in terms of organ at risk sparing except contralateral kidney.[14] Cai et al., compared helical tomotherapy to VMAT and reported that using VMAT reduced mean kidney doses by 34\% (17.4 Gy vs $11.5 \mathrm{~Gy}$ ) with the advantage of lower monitor units and overall treatment times.[13] Also for locally advanced pancreas cancer, VMAT was shown to achieve similar dose levels for right kidney and significantly reduce doses for left kidney compared to 5 field IMRT.[12] In our analysis, VMAT plans revealed better overall sparing for right kidney and less amount of high dose regions for left kidney while no statistical difference was found in terms of mean dose and low dose areas for left kidney.

Similar to our study design, Ali et al., analyzed 10 patients' dosimetric data and concluded that using double arc VMAT for curative or adjuvant radiotherapy for pancreas cancer patients, provides lower mean and V20-V15 doses of both kidney compared to IMRT, but other organ doses of both plans were found to be similar.[22] This gain for bilateral kidneys was ranging from $14 \%-75 \%$. In another similar dosimetric study for evaluating the use of double arc VMAT for locally advanced pancreas tumors, reduced monitor units, bilateral kidney V15 dose, mean dose of liver, stomach and small bowel dose reported.[12] Kidney dose reduction was common in all the studies. In contrast to second study, high dose regions of liver and small bowel were reduced by VMAT in our study.

Respiration-induced movement of the upper abdominal organs such as pancreas, liver and kidneys were assessed in several studies and the largest movements were noticed in the cranio-caudal direction for pancreas and liver $(23.7 \pm 15.9 \mathrm{~mm}$ and $24.4 \pm 16.4 \mathrm{~mm}$ ) by Bussels et al.,[23] As tumor and normal organs than expected, to provide $99 \%$ geometric coverage, margins of $20 \mathrm{~mm}$ inferiorly, $10 \mathrm{~mm}$ anteriorly, $7 \mathrm{~mm}$ superiorly, and 4 $\mathrm{mm}$ posteriorly were advised.[24] In the planning of a radiation treatment for pancreatic cancer, to overcome the effect of movement, using 4D CT found to be an increasingly attractive factor for the efficacy of IMRT to reduce normal organ doses.[20] In addition to the novel techniques, respiratory-gated radiotherapy in end-ex- piration or end-inspiration was found to have limited benefits in sparing normal organs.[20] Sangalli et al., studied the impact of 4DCT planning for unresectable pancreatic cancer patients. The results revealed that by using 4DCT, target volumes reduced by $37 \%$ compared to standard target delineation.[25] In our protocol, 4D CT simulation was performed as a standard clinic routine for planning of pancreas cancer patients.

\section{Conclusions}

In the dosimetric comparison of ssIMRT and VMAT techniques based on 4D CT scans of postoperative pancreas cancer patients, both plans reach dosimetric organ goals, but VMAT provided superior dose distribution in terms of organs at risk such as bilateral kidneys and small intestine, as well as liver with reduced high dose volumes in this cohort without compromising CTV coverage. This study is limited by a relatively small number of patient's 4DCT scans but these results could be promising for more tolerable concurrent radiochemotherapy treatments and increasingly usage of VMAT for pancreas cancer as routine like the other sites such as head and neck.

\section{Disclosure Statement}

The authors declare no conflicts of interest.

\section{References}

1. Herreros-Villanueva M, Hijona E, Cosme A, Bujanda L. Adjuvant and neoadjuvant treatment in pancreatic cancer. World J Gastroenterol 2012;18(14):1565-72.

2. Macdonald JS, Smalley SR, Benedetti J, Hundahl SA, Estes NC, Stemmermann GN, et al. Chemoradiotherapy after surgery compared with surgery alone for adenocarcinoma of the stomach or gastroesophageal junction. N Engl J Med 2001;345(10):725-30.

3. Intensity Modulated Radiation Therapy Collaborative Working Group. Intensity-modulated radiotherapy: current status and issues of interest. Int J Radiat Oncol Biol Phys 2001;51(4):880-914.

4. Milano MT, Chmura SJ, Garofalo MC, Rash C, Roeske JC, Connell PP, et al. Intensity-modulated radiotherapy in treatment of pancreatic and bile duct malignancies: toxicity and clinical outcome. Int J Radiat Oncol Biol Phys 2004;59(2):445-53.

5. Landry JC, Yang GY, Ting JY, Staley CA, Torres W, Esiashvili N, et al. Treatment of pancreatic cancer tumors with intensity-modulated radiation therapy (IMRT) 
using the volume at risk approach (VARA): employing dose-volume histogram (DVH) and normal tissue complication probability (NTCP) to evaluate small bowel toxicity. Med Dosim 2002;27(2):121-9.

6. Ben-Josef E, Shields AF, Vaishampayan U, Vaitkevicius V, El-Rayes BF, McDermott P, et al. Intensity-modulated radiotherapy (IMRT) and concurrent capecitabine for pancreatic cancer. Int J Radiat Oncol Biol Phys 2004;59(2):454-9.

7. Brown MW, Ning H, Arora B, Albert PS, Poggi M, Camphausen $\mathrm{K}$, et al. A dosimetric analysis of dose escalation using two intensity-modulated radiation therapy techniques in locally advanced pancreatic carcinoma. Int J Radiat Oncol Biol Phys 2006;65(1):274-83.

8. https://www.rtog.org/CoreLab/ContouringAtlases/ PancreasAtlas.aspx. Accessed June 8, 2017.

9. Bittner MI, Grosu AL, Brunner TB. Comparison of toxicity after IMRT and 3D-conformal radiotherapy for patients with pancreatic cancer - a systematic review. Radiother Oncol 2015;114(1):117-21.

10. Kuo YC, Chiu YM, Shih WP, Yu HW, Chen CW, Wong PF, et al. Volumetric intensity-modulated Arc (RapidArc) therapy for primary hepatocellular carcinoma: comparison with intensity-modulated radiotherapy and 3-D conformal radiotherapy. Radiat Oncol 2011;6:76.

11. Fung-Kee-Fung SD, Hackett R, Hales L, Warren G, Singh AK. A prospective trial of volumetric intensitymodulated arc therapy vs conventional intensity modulated radiation therapy in advanced head and neck cancer. World J Clin Oncol 2012;3(4):57-62.

12. Eppinga W, Lagerwaard F, Verbakel W, Slotman B, Senan S. Volumetric modulated arc therapy for advanced pancreatic cancer. Strahlenther Onkol 2010;186(7):382-7.

13. Cai J, Yue J, McLawhorn R, Yang W, Wijesooriya K, Dunlap NE, et al. Dosimetric comparison of $6 \mathrm{MV}$ and $15 \mathrm{MV}$ single arc rapidarc to helical TomoTherapy for the treatment of pancreatic cancer. Med Dosim 2011;36(3):317-20.

14. Vieillot S, Azria D, Riou O, Moscardo CL, Dubois $\mathrm{JB}$, Aillères $\mathrm{N}$, et al. Bilateral kidney preservation by volumetric-modulated arc therapy (RapidArc) compared to conventional radiation therapy (3D-CRT) in pancreatic and bile duct malignancies. Radiat Oncol 2011;6:147.

15. Allen AM, Zalupski MM, Robertson JM, Eckhauser FE, Simone D, Brown D, et al. Adjuvant therapy in pancreatic cancer: Phase I trial of radiation dose escalation with concurrent full-dose gemcitabine. Int J Radiat Oncol Biol Phys 2004;59(5):1461-7.

16. Moertel CG, Frytak S, Hahn RG, O'Connell MJ, Reitemeier RJ, Rubin J, et al. Therapy of locally unresectable pancreatic carcinoma: a randomized comparison of high dose (6000 rads) radiation alone, moderate dose radiation (4000 rads + 5-fluorouracil), and high dose radiation + 5-fluorouracil: The Gastrointestinal Tumor Study Group. Cancer 1981;48(8):1705-10.

17. Regine WF, Winter KA, Abrams RA, Safran H, Hoffman JP, Konski A, et al. Fluorouracil vs gemcitabine chemotherapy before and after fluorouracil-based chemoradiation following resection of pancreatic adenocarcinoma: a randomized controlled trial. JAMA 2008;299(9):1019-26.

18. Yovino S, Poppe M, Jabbour S, David V, Garofalo M, Pandya N, et al. Intensity-modulated radiation therapy significantly improves acute gastrointestinal toxicity in pancreatic and ampullary cancers. Int J Radiat Oncol Biol Phys 2011;79(1):158-62.

19. Baglan KL, Frazier RC, Yan D, Huang RR, Martinez AA, Robertson JM. The dose-volume relationship of acute small bowel toxicity from concurrent 5-FUbased chemotherapy and radiation therapy for rectal cancer. Int J Radiat Oncol Biol Phys 2002;52(1):176-83.

20. van der Geld YG, van Triest B, Verbakel WF, van Sörnsen de Koste JR, Senan S, Slotman BJ, et al. Evaluation of four-dimensional computed tomography-based intensity-modulated and respiratory-gated radiotherapy techniques for pancreatic carcinoma. Int J Radiat Oncol Biol Phys 2008;72(4):1215-20.

21. Bahl A, Kapoor R, Tomar P, Singh OA, Gupta R, Sharma SC. Dosimetric comparison of doses to organs at risk using 3-D conformal radiotherapy versus intensity modulated radiotherapy in postoperative radiotherapy of periampullary cancers: implications for radiation dose escalation. JOP 2013;14(1):39-43.

22. Ali AN, Dhabaan AH, Jarrio CS, Siddiqi AK, Landry JC. Dosimetric comparison of volumetric modulated arc therapy and intensity-modulated radiation therapy for pancreatic malignancies. Med Dosim $2012 \mathrm{Au}$ tumn;37(3):271-5.

23. Bussels B, Goethals L, Feron M, Bielen D, Dymarkowski S, Suetens P, et al. Respiration-induced movement of the upper abdominal organs: a pitfall for the three-dimensional conformal radiation treatment of pancreatic cancer. Radiother Oncol 2003;68(1):69-74.

24. Feng M, Balter JM, Normolle D, Adusumilli S, Cao Y, Chenevert TL, et al. Characterization of pancreatic tumor motion using cine MRI: surrogates for tumor position should be used with caution. Int J Radiat Oncol Biol Phys 2009;74(3):884-91.

25. Sangalli G, Passoni P, Cattaneo GM, Broggi S, Bettinardi V, Reni M, et al. Planning design of locally advanced pancreatic carcinoma using 4DCT and IMRT/ IGRT technologies. Acta Oncol 2011;50(1):72-80. 